\title{
Linear fractional differential equations and eigenfunctions of fractional differential operators
}

\author{
Eliana Contharteze Grigoletto ${ }^{1}$ D . Edmundo Capelas de Oliveira ${ }^{2}$. \\ Rubens de Figueiredo Camargo ${ }^{3}$
}

Received: 4 May 2016 / Revised: 14 August 2016 / Accepted: 19 August 2016 /

Published online: 29 August 2016

(C) SBMAC - Sociedade Brasileira de Matemática Aplicada e Computacional 2016

\begin{abstract}
Eigenfunctions associated with Riemann-Liouville and Caputo fractional differential operators are obtained by imposing a restriction on the fractional derivative parameter. Those eigenfunctions can be used to express the analytical solution of some linear sequential fractional differential equations. As a first application, we discuss analytical solutions for the so-called fractional Helmholtz equation with one variable, obtained from the standard equation in one dimension by replacing the integer order derivative by the Riemann-Liouville fractional derivative. A second application consists of an initial value problem for a fractional wave equation in two dimensions in which the integer order partial derivative with respect to the time variable is replaced by the Caputo fractional derivative. The classical Mittag-Leffler functions are important in the theory of fractional calculus because they emerge as solutions of fractional differential equations. Starting with the solution of a specific fractional differential equation in terms of these functions, we find a way to express the exponential function in terms of classical Mittag-Leffler functions. A remarkable characteristic of this relation is that it is true for any value of the parameter $n$ appearing in the definition of the functions, i.e.,
\end{abstract}

Communicated by Antonio José Silva Neto.

RFC thanks CNPq-National Counsel of Technological and Scientific Development (455920/2014-1).

Eliana Contharteze Grigoletto

elianac@fca.unesp.br

Edmundo Capelas de Oliveira

capelas@ime.unicamp.br

Rubens de Figueiredo Camargo

rubens@fc.unesp.br

1 Departamento de Bioprocessos e Biotecnologia, FCA-UNESP, Rua José Barbosa de Barros 1780, Botucatu, SP 18610-307, Brazil

2 Departamento de Matemática Aplicada, IMECC-UNICAMP, Campinas, SP 13083-859, Brazil

3 Departamento de Matemática, Faculdade de Ciências, UNESP, Av. Eng. Luiz Edmundo Carrijo Coube, 14-01 Bairro: Vargem Limpa, Bauru, SP 17033-360, Brazil 
we have an infinite family of different expressions for $e^{x}$ in terms of classical Mittag-Leffler functions.

Keywords Riemann-Liouville derivatives · Caputo derivatives · Linear fractional differential equations $\cdot$ Mittag-Leffler functions

Mathematics Subject Classification 26A33 Fractional derivatives and integrals

\section{Introduction}

The Non-Integer Order Calculus, traditionally known as Fractional Calculus (FC), is the branch of mathematics that deals with the study of integrals and derivatives of non-integer order. Although it is not accurate, since the order of an integral and a derivative can be real and also complex (Bagley and Torvik 1983; Tenreiro Machado et al. 2010a, b, 2011), it has played an outstanding role (Tenreiro Machado et al. 2011) since its creation, as several mathematicians and applied researchers have obtained important results by modeling real processes using FC (Arafa et al. 2016; Camargo et al. 2009a, 2012; Camargo and de Oliveira 2015; Debnath 2003; Mainardi 2009; Ortigueira and Tenreiro Machado 2015; Podlubny 1999; Soubhia et al. 2010).

Given a differential equation that describes a specific phenomenon, a common way to use fractional modeling is to replace the integer order derivatives by non-integer derivatives, usually with order lower than or equal to the order of the original derivatives, so that the usual solutions may be recovered as a particular case (Camargo and de Oliveira 2015).

Although there is no trivial physical and geometrical interpretation for the fractional derivative and the fractional integral (Podlubny 2002; Tavassoli et al. 2013), fractional order differential equations are naturally related to systems with memory, as fractional derivatives are usually nonlocal operators, i.e., the calculation of a time-fractional derivative at a given time requires its knowledge at all previous times (Camargo and de Oliveira 2015; Podlubny 1999). Processes with memory exist in many biological systems (Arafa et al. 2016; Diethelm et al. 2005; Elsadany and Matouk 2014; Matouk et al. 2015; El-Sayed et al. 2009, 2007; Arafa et al. 2012). Besides, fractional differential equations may help in reducing the errors arising from the neglected parameters in modeling real-life phenomena (Arafa et al. 2016; Mainardi 2009; Gutierrez et al. 2010).

There are several applications of fractional calculus in engineering (Sabatier et al. 2007), for example, in the study of control and dynamical systems (Matignon 1996; Matouk 2010, 2015). Moreover, there are in physics several potential applications of fractional derivatives (Hilfer 2000), for instance, in the generalization of classical equations (Camargo et al. 2008, 2009a, b, c).

In medicine, it has been found that the electrical conductance of the cell membranes of living organisms are described by fractional order equations, so that they may be classified as groups of non-integer order models. Fractional derivatives embody essential features of cell rheological behavior and have enjoyed a great success in the field of rheology (Arafa et al. 2016). Some mathematical models in HIV show that fractional models are more approximate than their integer order versions (Arafa et al. 2016; Diethelm et al. 2005).

With the aim of solving fractional partial differential equations and generalizing results, several definitions of "fractional derivative" (FD) have been proposed (de Oliveira and Tenreiro Machado 2014). In a recent paper (Khalil et al. 2014), a new definition of FD, called conformable FD, has also been proposed. Another example is the so-called local FD 
(Kolwankar and Gangal 1996). Finally, one should also consider the Grünwald-Letnikov, Riemann-Liouville and Caputo fractional derivatives and the Riesz potential. A natural question arises: "What is a fractional derivative?" In a paper whose title is exactly this question (Ortigueira and Tenreiro Machado 2015), Ortigueira and Tenreiro Machado set a criterion named Wide Sense Criterion (WSC) which establishes when an operator is an FD and showed that the well-known definitions of Grünwald-Letnikov, Riemann-Liouville, Caputo and Riesz satisfy the WSC.

Since linear sequential fractional differential equations are naturally related to the modeling of many problems in physics and applied science, several methods to obtain the analytical solutions for this kind of equations have been studied (Bonilla et al. 2005; Dzherbashyan and Nersesyan 1968; Kilbas et al. 2006, 2007; Miller and Ross 1993; Rida and Arafa 2011).

This work presents, with the help of some particular linear fractional differential equations, new theorems about eigenfunctions related to the fractional differential operators of Riemann-Liouville, $D_{a+}^{n \alpha}$ and of Caputo ${ }^{\mathrm{C}} D_{a+}^{n \alpha}$. From these theorems, we may express the exponential function in terms of a sum involving the Mittag-Leffler functions (Grigoletto 2014).

The results are presented as follows: after this introduction, Sect. 2 presents a review of FC and some preliminary concepts. In Sect. 3, the definition of the so-called linear sequential fractional differential equation and new theorems are introduced. In Sect. 4, we present the analytical solution of some particular linear sequential fractional differential equations. Finally, in Sect. 5, a new formula involving the exponential and the Mittag-Leffler functions is presented. Section 6 brings the concluding remarks.

\section{Preliminary concepts}

In this section, we present several definitions and properties of the Riemann-Liouville fractional integral, the Riemann-Liouville fractional derivative and the Caputo fractional derivatives.

In what follows, for Eqs. (1-5), we assume that $\alpha>0, \Omega=(a, b)$ is an interval on the real axis $\mathbb{R}=(-\infty, \infty)$ and $f \in \mathrm{C}^{n}[a, b]$, where $^{1} n=[\alpha]+1$.

The Riemann-Liouville left-sided fractional integral of order $\alpha$, denoted $I_{a+}^{\alpha}$, is defined as follows:

$$
\left(I_{a+}^{\alpha} f\right)(x):=\frac{1}{\Gamma(\alpha)} \int_{a}^{x} \frac{f(\tau)}{(x-\tau)^{1-\alpha}} \mathrm{d} \tau, \quad \text { for } x \in \Omega,
$$

where

$$
\Gamma(\alpha)=\int_{0}^{\infty} t^{\alpha-1} e^{-t} \mathrm{~d} t
$$

is the Gamma function (de Oliveira 2005).

For convenience, $I_{a+}^{0}$ is considered as the identity operator.

The Riemann-Liouville left-sided fractional derivative of order $\alpha$, denoted $D_{a+}^{\alpha}$, is defined as follows:

$$
\left(D_{a+}^{\alpha} f\right)(x):=\frac{\mathrm{d}^{n}}{\mathrm{~d} x^{n}}\left[\left(I_{a+}^{n-\alpha} f\right)(x)\right], \quad \text { for } \quad x \in \Omega .
$$

$1[\mu]$ indicates the integer part of $\mu$. 
In particular, if $0<\alpha<1$ we may write from Eq. (3) that

$$
\left(D_{a+}^{\alpha} f\right)(x)=\left(\frac{\mathrm{d}}{\mathrm{d} x}\right)\left[\left(I_{a+}^{1-\alpha} f\right)(x)\right]=\frac{1}{\Gamma(1-\alpha)}\left(\frac{\mathrm{d}}{\mathrm{d} x}\right) \int_{a}^{x} \frac{f(\tau)}{(x-\tau)^{\alpha}} \mathrm{d} \tau .
$$

If $\alpha=1$, Eq. (3) becomes $\left(D_{a+}^{1} f\right)(x)=f^{\prime}(x)$.

Now we present Caputo's definition for the fractional derivative (Mainardi 2009).

The Caputo left-sided fractional derivative of order $\alpha$, denoted by $\mathrm{C}_{D_{a+}^{\alpha}}^{\alpha}$, is defined as follows:

$$
\left(\mathrm{C}_{D_{a+}^{\alpha}}^{\alpha}\right)(x):=\left(I_{a+}^{n-\alpha} f^{(n)}\right)(x), \text { for } x \in \bar{\Omega} .
$$

As a particular case, if $0<\alpha<1$, Eq. (5) becomes

$$
\left({ }^{\mathrm{C}} D_{a+}^{\alpha} f\right)(x):=\left(I_{a+}^{1-\alpha} f^{\prime}\right)(x)=\frac{1}{\Gamma(1-\alpha)} \int_{a}^{x} \frac{f^{\prime}(\tau)}{(x-\tau)^{\alpha}} \mathrm{d} \tau .
$$

If $\alpha=1$, we obtain from Eq. (5) that $\left(\mathrm{C}_{D_{a+}^{1}}^{1} f\right)(x)=f^{\prime}(x)$.

Property 1 Let $\Omega=(a, b)$ be an interval on the real axis $\mathbb{R}, \mu>-1$ and $\alpha>0$; then

$$
D_{a+}^{\alpha}(x-a)^{\mu}=\frac{\Gamma(\mu+1)}{\Gamma(\mu-\alpha+1)}(x-a)^{\mu-\alpha} \quad \text { for } \quad x \in \Omega \text {. }
$$

Property 2 Let $\Lambda=[a, b]$ be an interval on the real axis $\mathbb{R}$ and $\alpha>0$. Then, for $x \in \Lambda$ we have

$$
{ }^{\mathrm{C}} D_{a+}^{\alpha}(x-a)^{\mu}= \begin{cases}0, & \text { for } \mu=0,1,2, \ldots,[\alpha], \\ \frac{\Gamma(\mu+1)}{\Gamma(\mu-\alpha+1)}(x-a)^{\mu-\alpha}, & \text { for } \mu>[\alpha] .\end{cases}
$$

\subsection{Mittag-Leffler functions}

The Mittag-Leffler functions are generalizations of the exponential function. They play in FC a role similar to the role played by the exponential function in usual calculus.

The classical Mittag-Leffler function is defined as (Mittag-Leffler 1903):

$$
E_{\alpha}(z)=\sum_{j=0}^{\infty} \frac{z^{j}}{\Gamma(\alpha j+1)}, \quad z \in \mathbb{C} \quad \text { and } \mathcal{R}(\alpha)>0,
$$

where $\mathcal{R}(\gamma)$ denotes the real part of $\gamma$. Taking $\alpha=1$ in Eq. (8), the exponential function is recovered because $E_{1}(z)=\mathrm{e}^{z}$. A two-parameter generalization has been proposed by Wiman (Wiman 1905) as follows:

$$
E_{\alpha, \beta}(z)=\sum_{j=0}^{\infty} \frac{z^{j}}{\Gamma(\alpha j+\beta)}, \quad \text { with } \mathcal{R}(\alpha)>0 \quad \text { and } \mathcal{R}(\beta)>0 .
$$

For $\beta=1$, the classical Mittag-Leffler function is recovered because $E_{\alpha, 1}(z)=E_{\alpha}(z)$.

The Caputo fractional derivative of the classical Mittag-Leffler function is given by

$$
\mathrm{C}_{D_{0+}^{\alpha}\left[E_{\alpha}\left(z^{\alpha}\right)\right]=E_{\alpha}\left(z^{\alpha}\right) .}
$$

This shows that in the case of the Caputo fractional derivative, the Mittag-Leffler function recovers the most important property of the exponential function. That is the reason why some authors refer to Mittag-Leffler functions as the fractional generalization of the exponential function. 


\section{Linear fractional differential equations}

In this paper, we employ the left-sided fractional operators explained above. A similar reasoning will extend the results obtained here to right-sided fractional operators.

Let $\Lambda=[a, b]$ be an interval on the real axis $\mathbb{R}, x \in \Lambda, 0<\alpha \leq 1, n \in \mathbb{N}$ and $g, a_{j}: \Lambda \rightarrow \mathbb{R}$ continuous functions for $j=0,1,2, \ldots, n-1$. The linear sequential fractional differential equations (Kilbas et al. 2006, 2007; Rida and Arafa 2011) of order $n \alpha$, denoted by $\left[\mathrm{L}_{n \alpha}(f)\right](x)$, are defined as

$$
\left[\mathrm{L}_{n \alpha}(f)\right](x):=\left(D_{a+}^{n \alpha} f\right)(x)+\sum_{j=0}^{n-1} a_{j}(x)\left(D_{a+}^{j \alpha} f\right)(x)=g(x),
$$

where $D_{a+}^{\alpha}$ represents the fractional derivative of Riemann-Liouville (or Caputo) and

$$
\left(D_{a+}^{0} f\right)(x)=f^{(0)}(x)=f(x) .
$$

In this paper, we consider the particular case in which $a_{0}(x)=\lambda$, with $\lambda \neq 0, a_{j}(x)=0$, for $j=1,2, \ldots, n-1$ and $g(x)=0$ in Eq. (11). As a result, we obtain a homogeneous linear sequential fractional differential equation, denoted by $\left[\hat{\mathrm{L}}_{n \alpha}(f)\right](x)$ and defined as

$$
\left[\hat{\mathrm{L}}_{n \alpha}(f)\right](x):=\left(D_{a+}^{n \alpha} f\right)(x)-\lambda f(x)=0 .
$$

From the two theorems presented below about eigenfunctions of the Riemann-Liouville and Caputo fractional operators it is possible to find solutions for fractional differential equations of the type of Eq. (12), where $D_{a+}^{\alpha}$ can be the Riemann-Liouville or the Caputo fractional operators.

Theorem 1 Let $\Omega=(a, b)$ be an interval on the real axis $\mathbb{R}, n \in \mathbb{N}, \lambda \neq 0$ a real number and $\alpha \in\left(\frac{n-1}{n}, 1\right]$ an interval on the real axis. Then

$$
f_{k}(x)=(x-a)^{n \alpha-k} E_{n \alpha, n \alpha+1-k}\left[\lambda(x-a)^{n \alpha}\right],
$$

for $x \in \Omega$ and $k=1,2, \ldots, n$, are the eigenfunctions associated with the eigenvalues $\lambda$ of the Riemann-Liouville left-sided fractional derivative $D_{a+}^{n \alpha}$.

Proof For any fixed value $k, k=1,2, \ldots, n$, we may write, from Eq. (9),

$$
\begin{aligned}
(x-a)^{n \alpha-k} E_{n \alpha, n \alpha+1-k}\left[\lambda(x-a)^{n \alpha}\right] & =\sum_{j=0}^{\infty} \frac{\lambda^{j}(x-a)^{n \alpha j+n \alpha-k}}{\Gamma(n \alpha j+n \alpha+1-k)} \\
& =\sum_{j=1}^{\infty} \frac{\lambda^{j-1}(x-a)^{n \alpha j-k}}{\Gamma(n \alpha j+1-k)} .
\end{aligned}
$$

Thus, we may write

$$
D_{a+}^{n \alpha}\left\{(x-a)^{n \alpha-k} E_{n \alpha, n \alpha+1-k}\left[\lambda(x-a)^{n \alpha}\right]\right\}=\sum_{j=1}^{\infty} \frac{\lambda^{j-1} D_{a+}^{n \alpha}\left[(x-a)^{n \alpha j-k}\right]}{\Gamma(n \alpha j+1-k)} .
$$

From Eq. (7) in Property 1 it follows that

$$
D_{a+}^{n \alpha}\left[(x-a)^{n \alpha j-k}\right]=\frac{\Gamma(n \alpha j-k+1)}{\Gamma(n \alpha j-k-n \alpha+1)}(x-a)^{n \alpha j-k-n \alpha}, \quad n \alpha j-k>-1,
$$


for $k \in\{1,2, \ldots, n\}$ and $j \in\{1,2,3, \ldots\}$. Now,

$$
n \alpha j-k>-1 \quad \Longrightarrow \quad \alpha>\frac{k-1}{n j} \text {. }
$$

Since $\alpha \in\left(\frac{n-1}{n}, 1\right]$, all inequalities in Eq. (16) are verified.

Using the result of Eq. (15) in Eq. (14), we obtain

$$
D_{a+}^{n \alpha}\left\{(x-a)^{n \alpha-k} E_{n \alpha, n \alpha+1-k}\left[\lambda(x-a)^{n \alpha}\right]\right\}=\sum_{j=1}^{\infty} \frac{\lambda^{j-1}(x-a)^{n \alpha j-k-n \alpha}}{\Gamma(n \alpha j-k-n \alpha+1)} .
$$

If $j=1$, then the term

$$
\frac{(x-a)^{-k}}{\Gamma(1-k)},
$$

in the second member of Eq. (17) vanishes for $k \in\{1,2, \ldots, n\}$, since

$$
\lim _{t \rightarrow k} \frac{(x-a)^{-t}}{\Gamma(1-t)}=0
$$

for $k \in\{1,2, \ldots, n\}$. In consequence, Eq. (17) can be rewritten as

$$
\begin{aligned}
D_{a+}^{n \alpha}\left\{(x-a)^{n \alpha-k} E_{n \alpha, n \alpha+1-k}\left[\lambda(x-a)^{n \alpha}\right]\right\} & =\sum_{j=2}^{\infty} \frac{\lambda^{j-1}(x-a)^{n \alpha j-k-n \alpha}}{\Gamma(n \alpha j-k-n \alpha+1)} \\
& =\sum_{j=0}^{\infty} \frac{\lambda^{j+1}(x-a)^{n \alpha j+n \alpha-k}}{\Gamma(n \alpha j+n \alpha+1-k)} .
\end{aligned}
$$

Thus,

$D_{a+}^{n \alpha}(x-a)^{n \alpha-k} E_{n \alpha, n \alpha+1-k}\left[\lambda(x-a)^{n \alpha}\right]=\lambda(x-a)^{n \alpha-k} E_{n \alpha, n \alpha+1-k}\left[\lambda(x-a)^{n \alpha}\right]$,

for $\alpha \in\left(\frac{n-1}{n}, 1\right], x \in \Omega$ and $k=1,2, \ldots, n$, which is the expected result.

Now, considering the Caputo left-sided fractional derivative, we have the following theorem:

Theorem 2 Let $\Lambda=[a, b]$ be an interval on the real axis $\mathbb{R}, n \in \mathbb{N}, \lambda \neq 0$ a real number and $\alpha \in\left(\frac{n-1}{n}, 1\right]$ an interval on the real axis. Then

$$
\varphi_{k}(x)=(x-a)^{k-1} E_{n \alpha, k}\left[\lambda(x-a)^{n \alpha}\right],
$$

with $x \in \Lambda$ and $k=1,2, \ldots, n$, are the eigenfunctions associated with the eigenvalues $\lambda$ of the Caputo left-sided fractional derivative ${ }^{\mathrm{C}} D_{a+}^{n \alpha}$.

Proof For a fixed value $k$, we obtain from Eq. (9):

$$
(x-a)^{k-1} E_{n \alpha, k}\left[\lambda(x-a)^{n \alpha}\right]=\sum_{j=0}^{\infty} \frac{\lambda^{j}(x-a)^{n \alpha j+k-1}}{\Gamma(n \alpha j+k)} .
$$


Thus, we may write

$$
{ }^{\mathrm{C}} D_{a+}^{n \alpha}\left\{(x-a)^{k-1} E_{n \alpha, k}\left[\lambda(x-a)^{n \alpha}\right]\right\}=\sum_{j=0}^{\infty} \frac{\lambda^{j}}{\Gamma(n \alpha j+k)}{ }^{\mathrm{C}} D_{a+}^{n \alpha}\left[(x-a)^{n \alpha j+k-1}\right] .
$$

Since $\alpha \in\left(\frac{n-1}{n}, 1\right]$, we have

$$
k-1 \leq[n \alpha],
$$

for $k \in\{1,2, \ldots, n\}$, and

$$
n \alpha j+k-1>[n \alpha],
$$

for $k \in\{1,2, \ldots, n\}$ and $j \in\{1,2,3, \ldots\}$.

Using Eq. (21) and Property 2, it follows that

$$
{ }^{\mathrm{C}} D_{a+}^{n \alpha}(x-a)^{k-1}=0,
$$

for $k \in\{1,2, \ldots, n\}$. From Property 2 and Eq. (22),

$$
{ }^{\mathrm{C}} D_{a+}^{n \alpha}\left[(x-a)^{n \alpha j+k-1}\right]=\frac{\Gamma(n \alpha j+k)(x-a)^{n \alpha j+k-1-n \alpha}}{\Gamma(n \alpha j+k-n \alpha)},
$$

for $j \in\{1,2,3, \ldots\}$ and $k \in\{1,2, \ldots, n\}$.

Using results (23) and (24) in Eq. (20), it follows that

$$
\sum_{j=0}^{\infty} \frac{\lambda^{j}}{\Gamma(n \alpha j+k)}{ }^{\mathrm{C}} D_{a+}^{n \alpha}\left[(x-a)^{n \alpha j+k-1}\right]=\sum_{j=1}^{\infty} \frac{\lambda^{j}(x-a)^{n \alpha j+k-1-n \alpha}}{\Gamma(n \alpha j+k-n \alpha)} .
$$

Then, from Eq. (25), we have

$$
\sum_{j=0}^{\infty} \frac{\lambda^{j}}{\Gamma(n \alpha j+k)}{ }^{\mathrm{C}} D_{a+}^{n \alpha}\left[(x-a)^{n \alpha j+k-1}\right]=\sum_{j=0}^{\infty} \frac{\lambda^{j+1}(x-a)^{n \alpha j+k-1}}{\Gamma(n \alpha j+k)} .
$$

Substituting result (26) in (20), we may write

$$
{ }^{\mathrm{C}} D_{a+}^{n \alpha}\left\{(x-a)^{k-1} E_{n \alpha, k}\left[\lambda(x-a)^{n \alpha}\right]\right\}=\sum_{j=0}^{\infty} \frac{\lambda^{j+1}(x-a)^{n \alpha j+k-1}}{\Gamma(n \alpha j+k)},
$$

from where it follows the expected result,

$$
{ }^{\mathrm{C}} D_{a+}^{n \alpha}\left\{(x-a)^{k-1} E_{n \alpha, k}\left[\lambda(x-a)^{n \alpha}\right]\right\}=\lambda(x-a)^{k-1} E_{n \alpha, k}\left[\lambda(x-a)^{n \alpha}\right],
$$

for $\alpha \in\left(\frac{n-1}{n}, 1\right], x \in \Lambda$ and $k=1,2, \ldots, n$.

The results of Theorems 1 and 2 for the corresponding right-sided fractional derivatives are proved similarly.

Proposition 1 If $\left\{y_{k}(x)\right\}_{k=1}^{n}$ is a fundamental system of solutions for the linear sequential fractional differential Eq. (11) with $g(x)=0$, then the general solution of the equation is given by

$$
y(x)=\sum_{k=1}^{n} c_{k} y_{k}(x),
$$

where $\left\{c_{k}\right\}_{k=1}^{n}$ are arbitrary constants.

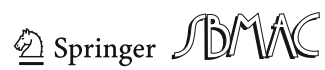


Proof See Kilbas et al. (2006).

Corollary 1 Let $\Omega=(a, b)$ be an interval on the real axis $\mathbb{R}, n$ a natural number and $\alpha \in$ $\mathbb{R}\left(\frac{n-1}{n}<\alpha \leq 1\right)$. Then the general solution of the linear sequential fractional differential equation

$$
\left(D_{a+}^{n \alpha} f\right)(x)-\lambda f(x)=0
$$

can be written as

$$
f_{\alpha}(x)=\sum_{k=1}^{n} c_{k}(x-a)^{n \alpha-k} E_{n \alpha, n \alpha+1-k}\left[\lambda(x-a)^{n \alpha}\right],
$$

where $\left\{c_{k}\right\}_{k=1}^{n}$ are arbitrary constants and $x \in \Omega$.

Proof From Theorem 1, the functions $\left\{f_{k}(x)\right\}_{k=1}^{n}$ satisfy the differential Eq. (30). As a result, from Proposition 1, it follows that the general solution of the equation can be written as

$$
f_{\alpha}(x)=\sum_{k=1}^{n} c_{k} f_{k}(x)
$$

Corollary 2 Let $\Lambda=[a, b]$ be an interval on the real axis $\mathbb{R}, n$ a natural number and $\alpha \in \mathbb{R}$, $\left(\frac{n-1}{n}<\alpha \leq 1\right)$. Then the general solution of the linear sequential differential equation

$$
\left({ }^{\mathrm{C}} D_{a+}^{n \alpha} \varphi\right)(x)-\lambda \varphi(x)=0
$$

can be written as

$$
\varphi_{\alpha}(x)=\sum_{k=1}^{n} c_{k}(x-a)^{k-1} E_{n \alpha, k}\left[\lambda(x-a)^{n \alpha}\right],
$$

where $\left\{c_{k}\right\}_{k=1}^{n}$ are arbitrary constants and $x \in \Lambda$.

Proof From Theorem 2, the functions $\left\{\varphi_{k}(x)\right\}_{k=1}^{n}$ satisfy Eq. (32). Then, from Proposition 1, it follows that the general solution of the differential equation can be written as

$$
\varphi_{\alpha}(x)=\sum_{k=1}^{n} c_{k} \varphi_{k}(x) \text {. }
$$

Remark 1 Note that, if $\alpha=1$, we have from Eqs. (31) and (33) that

$$
f_{1}(x)=\varphi_{1}(x)=\sum_{k=1}^{n} c_{k}(x-a)^{k-1} E_{n, k}\left[\lambda(x-a)^{n}\right] .
$$




\section{Differential equation $\left[\hat{\mathrm{L}}_{n \alpha}(f)\right](x)$}

There are recent applications of FC in different fields of knowledge (Baleanu et al. 2010; Dalir and Bashour 2010; Pfaffenzeller et al. 2011; Rahimy 2010; Silva Costa et al. 2015) and several methods have been proposed for solving fractional differential equations (Grigoletto and de Oliveira 2013; Elsaid 2010; Rajaramanr 2012; de la Hoz and Vadillo 2016).

Kilbas et al. (2007) present the analytical solution of a class of linear sequential fractional differential equations. In this section, we present some examples of equations arising from Eqs. (30) and (32).

Example 1 Let $\Omega=(0, b)$ be an interval on the real axis $\mathbb{R}$ and $\alpha \in \mathbb{R}\left(\frac{2}{3}<\alpha \leq 1\right)$. Using Corollary 1 , the solution of the fractional differential equation

$$
\left(D_{0+}^{3 \alpha} f\right)(x)-f(x)=0,
$$

can be written as

$$
f_{\alpha}(x)=c_{1} x^{3 \alpha-1} E_{3 \alpha, 3 \alpha}\left(x^{3 \alpha}\right)+c_{2} x^{3 \alpha-2} E_{3 \alpha, 3 \alpha-1}\left(x^{3 \alpha}\right)+c_{3} x^{3 \alpha-3} E_{3 \alpha, 3 \alpha-2}\left(x^{3 \alpha}\right),
$$

where $\left\{c_{k}\right\}_{k=1}^{3}$ are arbitrary constants and $x \in \Omega$.

Example 2 Let $\Lambda=[0, b]$ be an interval on the real axis $\mathbb{R}, \alpha \in \mathbb{R}\left(\frac{1}{2}<\alpha \leq 1\right)$, and $\lambda \neq 0$ a real constant. From Corollary 2, the solution of the following homogeneous fractional Helmholtz equation in one variable, which represents a time-independent form of the wave equation,

$$
\left(D_{0+}^{2 \alpha} \varphi\right)(x)+\lambda^{2} \varphi(x)=0,
$$

can be written as

$$
\varphi_{\alpha}(x)=c_{1} x^{2 \alpha-1} E_{2 \alpha, 2 \alpha}\left(-\lambda^{2} x^{2 \alpha}\right)+c_{2} x^{2 \alpha-2} E_{2 \alpha, 2 \alpha-1}\left(-\lambda^{2} x^{2 \alpha}\right),
$$

where $c_{1}$ and $c_{2}$ are arbitrary constants.

From Grigoletto (2014), we know that

$$
E_{2}(z)=\cosh (\sqrt{z}) \quad \text { and } \quad E_{2,2}(z)=\frac{\sinh \sqrt{z}}{\sqrt{z}} .
$$

Thus,

$$
E_{2}\left(-\lambda^{2} x^{2}\right)=\cosh (i|\lambda| x)=\cos (|\lambda| x)
$$

and

$$
E_{2,2}\left(-\lambda^{2} x^{2}\right)=\frac{\sinh (i|\lambda| x)}{i|\lambda| x}=\frac{\sin (|\lambda| x)}{|\lambda| x} .
$$

If $\alpha=1$ in Eq. (37), using Eqs. (39) and (40) in Eq. (38), we obtain a particular solution for the Helmholtz equation,

$$
\Delta \varphi+\lambda^{2} \varphi=0
$$

namely

$$
\varphi_{1}(x)=c_{1} \cos (|\lambda| x)+c_{2} \frac{\sin (|\lambda| x)}{|\lambda|},
$$

where $\left\{c_{k}\right\}_{k=1}^{2}$ are arbitrary constants and $x \in \Lambda$. 


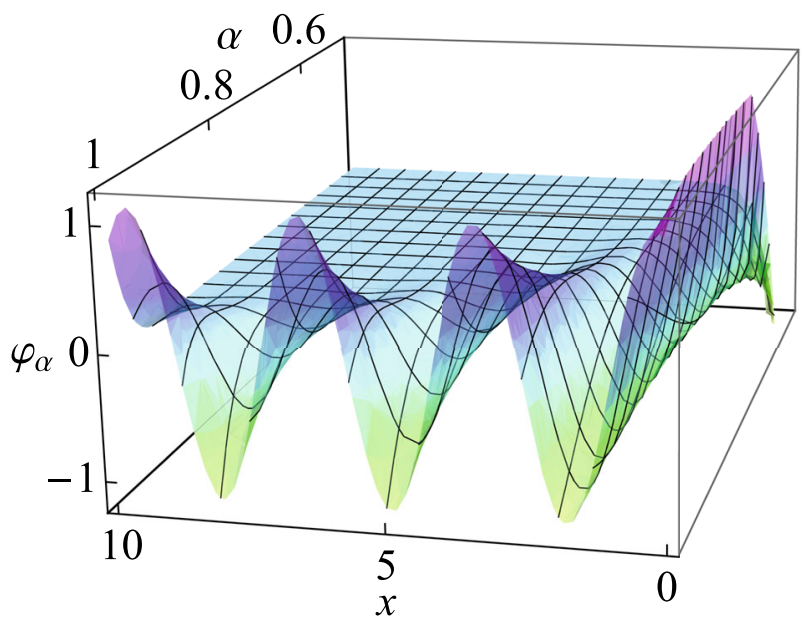

Fig. 1 Graphic for $\varphi_{\alpha}(x)=x^{2 \alpha-1} E_{2 \alpha, 2 \alpha}\left(-4 x^{2 \alpha}\right)+x^{2 \alpha-2} E_{2 \alpha, 2 \alpha-1}\left(-4 x^{2 \alpha}\right)$ with $\frac{1}{2}<\alpha \leq 1$

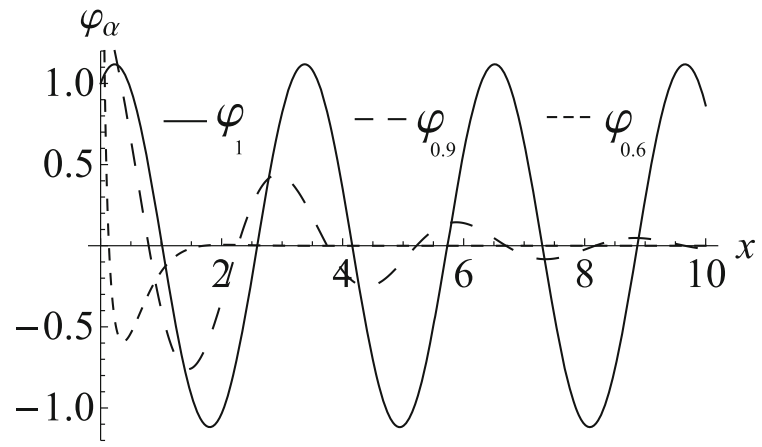

Fig. 2 Curves for $\varphi_{1}(x), \varphi_{0.9}(x)$ and $\varphi_{0.6}(x)$ when $x \in[0,10]$

The graphic of the solution given in Eq. (38) for different values of $\alpha\left(\frac{1}{2}<\alpha \leq 1\right)$ when $c_{1}=c_{2}=1$ and $\lambda=2$ is shown in Fig. 1. In Fig. 2, we plot the cases $\alpha=1, \alpha=0.9$ and $\alpha=0.6$.

Example 3 Let $u \equiv u(x, y, t)$ be a function defined on a space-time domain $(x, y, t) \in \Omega \times I$, where $\Omega \subseteq \mathbb{R}^{2}, I=[0, \mathrm{~T}]$ is an interval on the real axis $\mathbb{R}$ and $\alpha \in \mathbb{R}\left(\frac{1}{2}<\alpha \leq 1\right)$. Consider the initial value problem associated with the two-dimensional fractional wave differential equation

$$
\begin{cases}{ }^{\mathrm{C}} D_{0+}^{2 \alpha} u=\Delta u & \text { in } \quad(x, y, t) \in \Omega \times I, \\ u(x, y, 0)=\sin (x) \sin (y) & \text { in } \quad(x, y) \in \bar{\Omega}, \\ u_{t}(x, y, 0)=3 \sin (x) \sin (y) & \text { in } \quad(x, y) \in \bar{\Omega} .\end{cases}
$$

Let $u(x, y, t)=\psi(x, y) \phi(t)$, we then have

$$
\frac{{ }^{\mathrm{C}} D_{0+}^{2 \alpha} \phi(t)}{\phi(t)}=\frac{\Delta \psi(x, y)}{\psi(x, y)}=-\lambda^{2} .
$$


From Eq. (44), we have

$$
{ }^{\mathrm{C}} D_{0+}^{2 \alpha} \phi(t)=-\lambda^{2} \phi(t) .
$$

Now, using equation (38) in Example 2, we obtain the solution of Eq. (45),

$$
\phi(t)=c_{1} \cos \left(|\lambda| t^{\alpha}\right)+c_{2} t E_{2 \alpha, 2}\left(-\lambda^{2} t^{2 \alpha}\right),
$$

where $c_{1}$ and $c_{2}$ are arbitrary constants.

From Eq. (46) it follows that $\phi(0)=c_{1}$; as a result, using the initial condition $u(x, y, 0)=$ $\sin (x) \sin (y)$, we may write

$$
\psi(x, y)=\frac{1}{\phi(0)} \sin (x) \sin (y)=\frac{1}{c_{1}} \sin (x) \sin (y) .
$$

Thus, $\Delta \psi(x, y)=-2 \psi(x, y)$, and using this result in the equation

$$
\frac{\Delta \psi(x, y)}{\psi(x, y)}=-\lambda^{2}
$$

we have that $\lambda= \pm \sqrt{2}$.

So, the solution $u(x, y, t)$ can be written as

$$
u(x, y, t)=\sin (x) \sin (y)\left[\cos \left(\sqrt{2} t^{\alpha}\right)+c t E_{2 \alpha, 2}\left(-2 t^{2 \alpha}\right)\right],
$$

where $c$ is a real constant. Taking the derivative of $u$ with respect to $t$ in Eq. (48), and using the initial condition $u_{t}(x, y, 0)=3 \sin (x) \sin (y)$, we have that $c=3$, since $E_{2 \alpha, 2}(0)=1$.

As a result, the general solution of the two-dimensional wave fractional differential equation with the mentioned initial conditions is given by

$$
u(x, y, t)=\sin (x) \sin (y)\left[\cos \left(\sqrt{2} t^{\alpha}\right)+3 t E_{2 \alpha, 2}\left(-2 t^{2 \alpha}\right)\right],
$$

provided that $(x, y, t) \in \Omega \times I$.

\section{Exponential function and the Mittag-Leffler functions}

As a consequence of our previous results, we present now a particular sum involving the Mittag-Leffler functions.

Starting from the theorems and corollaries presented in the third section, we use the solution of a particular differential equation to express the exponential function in terms of Mittag-Leffler functions.

If $\alpha=1=\lambda$, the linear sequential fractional differential equations presented in Corollaries 1 and 2 become the ordinary differential equation

$$
y^{(n)}(x)-y(x)=0,
$$

whose general solution when $c_{k}=1$, for $k=1,2, \ldots, n$, can be written as

$$
y_{n}(x)=\sum_{k=1}^{n} x^{k-1} E_{n, k}\left(x^{n}\right),
$$

with $x \in \Omega$.

The function $y(x)=e^{x}$ also satisfies Eq. (50), a fact that leads us to analyze the similarity between the exponential function and the function in Eq. (51). Indeed, we have the following result: 
Proposition 2 Let $\Omega=(0, b)$ be an interval on the real axis $\mathbb{R}$ and $n$ a natural number. Then the function

$$
\phi_{n}(x)=\sum_{k=1}^{n} x^{k-1} E_{n, k}\left(x^{n}\right),
$$

defined for $x \in \Omega$, satisfies the equation

$$
\phi_{n}(x)=e^{x},
$$

for any fixed natural number $n$. In particular,

$$
\sum_{k=1}^{\infty} x^{k-1} E_{n, k}\left(x^{n}\right)=e^{x}
$$

Proof It is known that

$$
\begin{aligned}
\phi_{n}(x) & =\sum_{k=1}^{n} x^{k-1} E_{n, k}\left(x^{n}\right) \\
& =E_{n, 1}\left(x^{n}\right)+x E_{n, 2}\left(x^{n}\right)+x^{2} E_{n, 3}\left(x^{n}\right)+\cdots+x^{n-1} E_{n, n}\left(x^{n}\right) .
\end{aligned}
$$

Writing each series for the Mittag-Leffler functions as in Eq. (9) we obtain

$$
\phi_{n}(x)=\sum_{j=0}^{\infty} \frac{x^{n j}}{\Gamma(n j+1)}+\sum_{j=0}^{\infty} \frac{x^{n j+1}}{\Gamma(n j+2)}+\cdots+\sum_{j=0}^{\infty} \frac{x^{n j+n-1}}{\Gamma(n j+n)},
$$

that is,

$$
\begin{aligned}
\phi_{n}(x)= & \underbrace{\left(1+\frac{x^{n}}{\Gamma(n+1)}+\frac{x^{2 n}}{\Gamma(2 n+1)}+\cdots\right)}_{\sum_{j=0}^{\infty} \frac{x^{n j}}{\Gamma(n j+1)}} \\
+ & \underbrace{\left(\frac{x}{\Gamma(2)}+\frac{x^{n+1}}{\Gamma(n+2)}+\frac{x^{2 n+1}}{\Gamma(2 n+2)}+\cdots\right)}_{\sum_{j=0}^{\infty} \frac{x^{n j+1}}{\Gamma(n j+2)}} \\
& +\underbrace{\left(\frac{x^{n-1}}{\Gamma(n)}+\frac{x^{2 n-1}}{\Gamma(2 n)}+\frac{x^{3 n-1}}{\Gamma(3 n)}+\cdots\right)}_{\sum_{j=0}^{\infty} \frac{x^{n j+n-1}}{\Gamma(n j+n)}} .
\end{aligned}
$$

Reorganizing the series terms we may write

$$
\phi_{n}(x)=1+\frac{x}{\Gamma(2)}+\cdots+\frac{x^{n-1}}{\Gamma(n)}+\frac{x^{n}}{\Gamma(n+1)}+\frac{x^{n+1}}{\Gamma(n+2)}+\cdots=\sum_{j=0}^{\infty} \frac{x^{j}}{j !}=e^{x},
$$

where $\Gamma(j+1)=j$ !. Thus, we have shown that 


$$
\sum_{k=1}^{n} x^{k-1} E_{n, k}\left(x^{n}\right)=e^{x},
$$

for any fixed natural number $n$. We may also recover the result of equation (56) through the inverse Laplace transform.

\section{Concluding remarks}

This work presents the so-called eigenfunctions associated with the Riemann-Liouville and Caputo fractional differential operators, considering the parameter $n \alpha$ with $n$ a natural number and $\alpha$ in a limited interval that depends on $n$. In both cases, the eigenfunctions were obtained in terms of the two-parameter Mittag-Leffler functions. Besides, the analytical solution of a particular homogeneous linear sequential fractional differential equations of the kind $\left[\hat{\mathrm{L}}_{n \alpha}(f)\right](x)$ was obtained through the eigenfunctions found in Theorems 1 and 2.

Examples of applications were discussed; in particular, the fractional differential equation associated with the two-dimensional wave equation was solved. As another application, a new expression involving the exponential function and the Mittag-Leffler functions was demonstrated.

Acknowledgements We are indebted to Dr. J. Emílio Maiorino for useful and fruitful discussions. We are grateful to the referees for a number of helpful suggestions that improved the manuscript. We also thank CNPq (455920/2014-1).

\section{References}

Arafa AAM, Hanafy IM, Gouda MI (2016) Stability analysis of fractional order HIV infection of ${ }^{+} \mathrm{T}$ cells with numerical solutions. J Fract Calc Appl 7(1):36-45

Arafa AAM, Rida SZ, Khalil M (2012) Fractional modeling dynamics of HIV and CD4+ T-cells during primary infection. Nonlinear Biomed Phys 6(1):1-7

Bagley RL, Torvik PJ (1983) A theoretical basis for the application of fractional calculus to viscoelasticity. J Rheol 27:201-210

Baleanu D, Güvenc ZB, Tenreiro Machado JA (2010) New trends in nanotechnology and fractional calculus applications. Springer, Dordrecht

Bonilla B, Rivero M, Trujillo JJ (2005) Theory of sequential linear differential equations. Application. Preprint, Departamento de Analysis Mathematico, Universidad de La Laguna

Camargo RF, de Oliveira EC (2015) Fractional calculus (in Portuguese). Editora Livraria da Física, São Paulo

Camargo RF, Chiacchio AO, de Oliveira EC (2008) Differentiation to fractional orders and the fractional telegraph equation. J Math Phys 49:033505

Camargo RF, Chiacchio AO, de Oliveira EC (2009) Solution of the fractional Langevin equation and the Mittag-Leffler functions. J Math Phys 50:063507

Camargo RF, de Oliveira EC, Vaz J Jr (2009) On anomalous diffusion and the fractional generalized Langevin equation for a harmonic oscillator. J Math Phys 50:123518

Camargo RF, Charnet R, Charnet R, de Oliveira EC (2009) On some fractional Green's functions. J Math Phys 50(043514):1-12

Camargo RF, de Oliveira EC, Vaz J Jr (2012) On the generalized Mittag-Leffler function and its application in a fractional telegraph equation. Math Phys Anal Geom 15(1):1-16

Dalir M, Bashour M (2010) Applications of fractional calculus. Appl Math Sci 4(21):1021-1032

de Oliveira EC (2005) Special functions and applications (in Portuguese). Editora Livraria da Física, São Paulo de Oliveira EC, Tenreiro Machado JA (2014) A review of definitions for fractional derivatives and integrals. Math Prob Eng 2014:1-6, Article ID 238459

de la Hoz F, Vadillo F (2016) Numerical simulations of time-dependent partial differential equations. J Comput Appl Math 295:175-184 
Debnath L (2003) Recent applications of fractional calculus to science and engineering. Int J Math Math Sci $54: 3413-3442$

Diethelm K, Ford NJ, Freed AD, Luchko Yu (2005) Algorithms for the fractional calculus: a selection of numerical methods. Comput Methods Appl Mech Eng 194:743-773

Dzherbashyan MM, Nersesyan AB (1968) Fractional derivatives and the Cauchy problem for differential equations of fractional order. Izv Akad Nauk Armajan SSR Ser Mater 3(1):3-29

Elsaid A (2010) The variational iteration method for solving Riesz fractional partial differential equations. Compt Math Appl 60:1940-1947

Elsadany AA, Matouk AE (2014) Dynamical behaviors of fractional-order Lotka-Volterra predator prey model and its discretization. J Appl Math Comput 49(1):269-283

El-Sayed AMA, El-Mesiry AEM, El-Saka HAA (2007) On the fractional-order logistic equation. Appl Math Lett 20:817-823

El-Sayed AMA, Rida SZ, Arafa AAM (2009) On the solutions of time-fractional bacterial chemotaxis in a diffusion gradient chamber. Int J Nonlinear Sci 7:485-492

Grigoletto EC (2014) Fractional differential equations and the Mittag-Leffler functions (in Portuguese). Doctoral Thesis, Imecc-Unicamp, Campinas

Grigoletto EC, de Oliveira EC (2013) Fractional versions of the fundamental theorem of calculus. Appl Math 4:23-33

Gutierrez RE, Rosario JM, Tenreiro Machado JA (2010) Fractional order calculus: basic concepts and engineering applications. Math Prob Eng 375858:19

Hilfer R (2000) Applications of fractional calculus in physics, 128th edn. World Scientific, Singapore

Khalil R, Al Horani M, Yousef A, Sababheh M (2014) A new definition of fractional derivative. J Comput Appl Math 264:65-70

Kilbas AA, Srivastava HM, Trujillo JJ (2006) Theory and applications of fractional differential equations. Elsevier, Amsterdam

Kilbas AA, Rivero M, Rodríguez-Germá L, Trujillo JJ (2007) $\alpha$-Analytic solutions of some linear fractional differential equations with variable coefficients. Appl Math Comput 187:239-249

Kolwankar KM, Gangal AD (1996) Fractional differentiability of nowhere differentiable functions and dimensions. Chaos 6:505

Mainardi F (2009) Fractional calculus and waves in linear viscoelasticity. Imperial College Press, London

Matignon D (1996) Stability results for fractional differential equations with applications to control processing. In: Proceedings of IMACS-SMC, vol 7. Lille, pp 963-968

Mittag-Leffler GM (1903) Sur la nouvelle fonction $E_{\alpha}(z)$. CR Acad Sci Paris 137:554-558

Matouk AE (2010) Dynamical behaviors, linear feedback control and synchronization of the fractional order Liu system. J Nonlinear Syst Appl 1(3):135-140

Matouk AE (2015) Chaos synchronization of a fractional-order modified Van der Pol-Duffing system via new linear control, backstepping control and Takagi Sugeno fuzzy approaches. Complexity. doi:10.1002/cplx. 21719

Matouk AE, Elsadany AA, Ahmed E, Agiza HN (2015) Dynamical behavior of fractional-order HastingsPowell food chain model and its discretization. Commun Nonlinear Sci Numer Simul 27:153-167

Miller KS, Ross B (1993) An introduction to the fractional calculus and fractional differential equations. Wiley, New York

Ortigueira MD, Tenreiro Machado JA (2015) What is a fractional derivative? J Comput Phys 293:4-13

Podlubny I (1999) Fractional differential equations. Academic Press, San Diego

Podlubny I (2002) Geometric and physical interpretation of fractional integration and fractional differentiation. Fract Calc Appl Anal 5:367-386

Pfaffenzeller RA, Lenzi MK, Lenzi EK (2011) Modeling of granular material mixing using fractional calculus. Int Rev Chem Eng 3(6):818-821

Rahimy M (2010) Applications of fractional differential equations. Appl Math Sci 4:2453-2461

Rajaramanr R (2012) Analytical solutions for the different forms of telegraph equations by homotopy analysis method. Glob J Sci Front Res 12(5):7-12

Rida SZ, Arafa AAM (2011) New method for solving linear fractional differential equations. Int J Differ Equ 2011:1-8, Article ID 814132

Sabatier J, Argrawal OP, Tenreiro Machado JA (2007) Advances in fractional calculus: theoretical developments and applications in physics and engineering. Springer, New York

Silva Costa F, Grigoletto EC, Vaz J Jr., de Oliveira EC (2015) Slowing-down of neutrons: a fractional model. Commun Appl Ind Math 6(2). doi:10.1685/journal.caim.538

Soubhia AL, Camargo RF, de Oliveira EC, Vaz J Jr (2010) Theorem for series in three-parameter Mittag-Leffler function. Fract Calc Appl Anal 13:9-20 
Tavassoli MH, Tavassoli A, Ostad Rahimi MR (2013) The geometric and physical interpretation of fractional order derivatives of polynomial functions. Differ Geom Dyn Syst 15:93-104

Tenreiro Machado JA, Kiryakova V, Mainardi F (2010a) A poster about the old history of fractional calculus. Fract Calc Appl Anal 13:447-454

Tenreiro Machado JA, Kiryakova V, Mainardi F (2010b) A poster about the recent history of fractional calculus. Fract Calc Appl Anal 13:329-334

Tenreiro Machado JA, Kiryakova V, Mainardi F (2011) Recent history of fractional calculus. Commun Nonlinear Sci Num Simul 16:1140-1153

Wiman A (1905) Über den fundamentalsatz in der teorie der functionen $\mathrm{E}_{\alpha}(x)$. Acta Math 29:191-201 